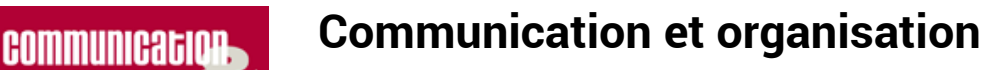

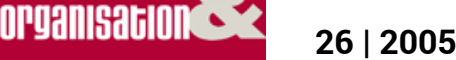

La communication des nouvelles éthiques de

l'entreprise

\title{
Vers une société des agendas. Une mutation de temporalités
}

\section{Gino Gramaccia}

\section{(2) OpenEdition}

\section{Journals}

Édition électronique

URL : http://journals.openedition.org/communicationorganisation/3303

DOI : 10.4000/communicationorganisation.3303

ISSN : $1775-3546$

\section{Éditeur}

Presses universitaires de Bordeaux

Édition imprimée

Date de publication : 2 janvier 2005

Pagination : 258-260

ISSN : $1168-5549$

\section{Référence électronique}

Gino Gramaccia, "Vers une société des agendas. Une mutation de temporalités », Communication et organisation [En ligne], 26 | 2005, mis en ligne le 19 juin 2012, consulté le 21 septembre 2020. URL : http://journals.openedition.org/communicationorganisation/3303; DOI : https://doi.org/10.4000/ communicationorganisation.3303

Ce document a été généré automatiquement le 21 septembre 2020.

(C) Presses universitaires de Bordeaux 


\title{
Vers une société des agendas. Une mutation de temporalités
}

\author{
Gino Gramaccia
}

1 L'auteur de l'Anthropologie des projets publie une vaste synthèse sur les problématiques contemporaines du temps, expliquant, dans une réflexion ample et nourrie de nombreuses références, les mutations des dynamiques sociales actuelles, marquées par la crise et l'urgence. L'action contemporaine est un processus qui doit être raconté selon des critères de rentabilité, d'utilité, d'efficacité et en fin de compte de temporalités contrôlées : "Ces temporalités opèrent délibérément un repli sur des horizons plus rapprochés que l'on peut aussi interpréter comme une nouvelle concentration inédite sur le seul moment présent » (p. 19). Vers quelle métrique du temps s'oriente alors le contrôle de ces processus hautement finalisés que sont les projets? L'auteur actualise la notion de synchroniseur pour désigner ces moments connectiques du temps, ces ajustements temporels entre des projets-processus de longueur et de complexité variables. L'agenda est donc un synchroniseur. Par coöncidence, au moment où j'écris ces lignes, je suis au Maroc. Le temps des souks, de la kasbah, le temps du thé, de la conversation, de l'écoute donne, par contraste, un relief bien singulier à l'économie du temps occidental dont on sait qu'elle calcule le temps de la présence, qu'elle évalue la pertinence des contacts entre des experts, qu'elle synchronise la part d'utilité de chacun dans les relations de travail. Ce temps de présence a donc un coût, puis un prix. Il peut signifier la négation d'Alter si Ego ne concède rien. Dans le souk, à l'inverse, il faut bien finir par concéder quelque chose, par donner quelque chose de soi-même. L'auteur prend soin de bien différencier temps et temporalité : la première expression désigne le temps objectivé de la mesure, la seconde, le temps vécu du sujet individuel ou collectif (p.37). Cette distinction prend un tour théorique plus précis lorsque l'auteur oppose, à propos des projets, deux modélisations du présent. Dans le Modèle Temporel 1 (MT1), «le présent devient le simple instrument permettant l'accès au futur » (p. 42) : le présent devient alors le vecteur fragile d'un futur désiré. Le Modèle Temporel 2 «nous introduit dans l'univers des temporalités plurielles» (p. 44): c'est toute la flèche du temps qui est tout entière mobilisée au service d'un présent, lequel prend alors le monopole du futur. La « temporalité dominante » des projets est une temporalité qui met l'accent sur le calcul 
de la disponibilité des ressources qu'offrent le passé et le futur. Ce présent accaparant requiert vigilance et responsabilité. Hans Jonas est cité. Les temporalités dominantes sont équivoques: émancipatrices, assujettissantes, ambiguës ou déniées, elles expriment surtout les contradictions insurmontables du monde contemporain dont les multiples projets de développement durable (p.50) font l'appoint, sans plus dans les argumentaires politiques.

Progressivement l'auteur nous invite à une méditation sur la signification du temps long, sur le sens du grand récit: la perspective d'un développement durable prolongeant le projet moderne d'une valorisation de l'histoire armerait notre patience et structurerait nos solidarités. A la contre-modernité du risque technologique se substituerait une modernité attachée à la production de savoirs réflexifs, des savoirs qui aident à la création des scénarios les plus favorables aux liens sociaux les plus solidaires: ceux qui s'inscrivent dans les temporalités les plus longues. Dans les nouvelles temporalités, la gestion anxieuse du risque s'est substituée à "l'avenir rayonnant de la prévision, de la prospective et de la futurologie (p. 83) et, pour tout dire, de la promesse. Mais la gestion s'assure au présent, provoquant ce que l'auteur appelle une "hypertrophie du moment présent» (p. 83), marque de la post-modernité. A cette conscience aiguë du présent, s'ajoute une pensée de la surface et de l'horizontalité. La post-modernité voit donc l'effondrement des systèmes dichotomiques (p. 99) (surface/ profondeur, jeunes/adultes, actifs/inactifs, image/référent... L'attachement postmoderne à la surface, c'est-à-dire à la simplicité de ce qui fixe rapidement le regard, signifie le détachement de la conscience vigilante aux choses instituées et par conséquent, nous y revenons, aux constructions de la mémoire et à ce qu'elles comptent préserver du futur. Dans le monde du travail, c'est le statut du salarié qui est à son tour déconstruit, détaché du lien de subordination pour devenir un "salariant ", comme on dit un « signifiant : acteur du discontinu, il contrôle des paramètres visibles, des tableaux de bord, des systèmes d'alerte et dans la foulée, s'affirme, par sa performance, comme responsable de sa propre sphère d'activité. Ce salariant marquerait le passage d'une "responsabilité émancipatrice à une responsabilité imposée " (p.103), d'une responsabilité de soi-même consécutive au délitement du lien de subordination. La compétence première d'un tel acteur est la vigilance: son environnement est celui d'un "projet-praxis» (p. 119) dont il construit autant le sens qu'il en subit les contraintes. Dans son histoire du paradigme du projet, l'auteur nous livre une précieuse intuition lorsque, à propos d'une crise dans l'art de construire qu'il date à la fin du Moyen Âge, il illustre la difficile convergence de l'idéal formel et du programme technique en prenant l'exemple de l'église Sancta Maria de Fiore à Florence : comment implanterun dôme sur un bâtiment déjà existant sans recourir à l'échafaudage? "Projet et complexité sont en étroite relation face à une situation-problème posée " (p.123). Comment résoudre la dualité qui oppose, d'une part, la traduction formelle de l'exigence architecturale telle qu'elle s'exprime par la schématisation, la maquette et de ce qui deviendra plus tard le prototype, et, d'autre part, la non disponibilité relative des ressources et des solutions dans l'environnement immédiat du constructeur? C'est par l'exploration et la combinaison de solutions par ailleurs et dans d'autres temps inventées que ce dépassement sera rendu possible. La vigilance technique ou la "veille » technologique parie sur la fécondité des connaissances aussi loin que porte le regard de l'historien-ingénieur. Le projet devient alors la méthodologie du recours à toutes les instances, hétérogènes, dispersées, disséminées dans un environnement complexe et incertain: ces instances sont des acteurs, des 
ressources, des moyens, des opportunités. Mais il convient surtout d'en régler l'ordonnancement selon le principe du "one shot». Au fond, pour les ressources, leur localisation spatiale est moins problématique que leur jalonnement sur la flèche du temps. Telle est la fonction de l'agenda comme paradigme technique de la synchronisation des temporalités : le téléphone cellulaire, les logiciels de planification, tous les systèmes informatiques de gestion de flux de données en sont des déclinaisons. Il faut toujours plus raccourcir le temps de la mise en œuvre opérationnelle de toujours plus de ressources en y ajoutant la fonction-clef de l'agenda : l'accord sur le point de simultanéité (la rencontre dans le temps) pour la gestion partagées de ces ressources. Cette question est duelle, bien sûr, dans le cas de l'agenda. Elle devient collective dans l'empilement des fonctions «agendaires» que constitue un planning. Les "figures actuelles de la simultanéité » sont l'expression de la rationalité des temporalités postmodernes : à chaque fois que possible, le gestionnaire doit s'efforcer d'éviter le partage non négociable des ressources, prévenir autant que faire se peut le surgissement d'aléas en aménageant des marges de liberté, des temporalités blanches destinées à résorber les effets de l'urgence.

Ce livre important, je l'ai lu dans un pays encore indifférent aux crises de l'urgence, aux figures de la simultanéité, aux croisements sans transcendance des "parties prenantes » sur des segments de temps. Mais pour combien de temps?

\section{AUTEUR}

\section{GINO GRAMACCIA}

De Jean-Pierre Boutinet, Paris, PUF, 2004, 256 p., $25 €$ 